\title{
Proportionally Fair Selective Cooperation for Cellular Networks: Algorithm, Simulation and Analysis ${ }^{\star}$
}

\author{
Erwu Liu ${ }^{1}$, Qinqing Zhang ${ }^{2}$, and Kin K. Leung ${ }^{1}$ \\ 1 Department of Electrical and Electronic Engineering, \\ Imperial College, London, UK, \\ \{erwu.liu, kin.leung\}@imperial.ac.uk \\ 2 Applied Physics Laboratory, Johns Hopkins University, USA, \\ Department of Computer Science, Johns Hopkins University, USA, \\ qinqing.zhang@jhuapl.edu
}

\begin{abstract}
We consider the problem of 2-node cooperation for cellular networks. In the considered system, a node and a cooperative relay are selected in a proportionally fair (PF) manner to transmit to the base station (BS), which uses the maximal ratio combining (MRC) for reception. The proposed scheme integrates the $\mathrm{PF}$ criterion in selective cooperation to maximize the overall utility of all 2-node cooperative transmissions, and is called proportionally fair selective cooperation (PFSC). This technique does not require distributed space-time coding algorithms and its simplicity allows for immediate implementation in existing hardware. In this research, we further provide a mathematical framework to analyze the performance of PFSC under a Rayleigh flat fading channel. We also present an analytical expression for quantifying the throughput gain of a PFSC-enabled cellular network over a traditional PFS-enabled cellular network without node cooperation. Using simulations, we show that our model is very accurate.

To our knowledge, it is the first time that a closed-form expression is obtained for the throughput of 2-node cooperative communication in a cellular network with the PF constraint.
\end{abstract}

Key words: Cooperative communications, proportional fairness

\section{Introduction}

In this work, we propose and analyze a practical cooperative scheme for cellular networks. The rationale for the extraordinary interest in exploiting cooperative techniques in wireless networks is very clear, as demonstrated by the high volume of publications in recent years [1-12]. Cooperative diversity [4] is a new form of diversity through distributed transmission and processing with node collaboration. Transmit cooperation has nodes exchanging each other's messages,

* This work was supported, in part, by Johns Hopkins University, Applied Physics Laboratory's internal research and development funds. 
sharing their antennas, and creating multiple paths to transmit the information. Receive cooperation has nodes forwarding information about their observations for decoding. A system with both transmit and receive cooperation is similar to a multiple-input multiple-output (MIMO) system in a networked manner. Therefore it is sometimes called a distributed MIMO or network MIMO [13]. In a network where each node is equipped with a single omnidirectional antenna, cooperative diversity can achieve similar gains from a MIMO system where each node is equipped with multiple antennas.

Researchers have proposed various network MIMO schemes for distributed wireless networks $[13,14]$. However, there are several limitations that hurdle the potential implementation of a network MIMO in a practical system compared to the conventional MIMO system. For example, synchronization among distributed antennas is much harder than a conventional MIMO transmitter. Additional resources such as bandwidth, power, time and complex code design are also required to enable the cooperation. Furthermore, antenna power allocation cannot be done as that in a conventional MIMO system, etc. However, these limitations and implementation complexity are significantly reduced in time-division-multiple-access (TDMA) cellular networks. Consequently, there are growing interests in cooperative diversity schemes for cellular networks. In this direction, this paper considers a practical 2-node cooperative scheme which requires minimum cooperation among the nodes for TMDA cellular networks and focuses on the throughput performance.

In a 2-node cooperative scenario for TDMA cellular networks, a Node $m$ together with a Node $r$ are opportunistically selected by the base station (BS) to transmit at next slot in a collaborative manner. Once the pair $(m, r)$ is scheduled, Node $m$ will transmit and Node $r$ will function as a decode-and-forward (DF) relay [4]. The BS will store the signal received from $m$ and use it together with the signal received from $r$ for successful decoding. Without node cooperation, the BS will only decode using the signal from $m$. With this scheme, better performance can be achieved as the BS will decode using the signals from two branches.

The BS can use various metrics to determine which $(m, r)$ pair will be scheduled to transmit. For example, to achieve the full spatial diversity order, selective relaying [6] can be extended to this 2-node cooperative scenario, i.e., only the best $(m, r)$ pair that provides the highest instantaneous capacity to the BS is selected at next slot. One can verify that, while this greedy policy provides the highest throughput performance of the whole system, it results in highly unfair resource utilization and will starve those nodes far away from the BS. Obviously, the performance of the considered system not only depends on node cooperation, it also depends on the metric used to select the $(m, r)$ pair. Originally from Kelly's work [15], the proportionally fair scheduling (PFS) algorithm [16-21] has spurred the development of a large number of network utility maximization (NUM) algorithms since 1997 [22-26], and is implemented in current 3G networks [27] as the most-cited NUM method. Being a promising scheme for fair resource allocation, PFS has shown excellent balance between throughput and fairness via multi-user diversity and game-theoretic equilibrium. In light of this, 
we apply the proportionally fair (PF) criterion in selecting the pair $(m, r)$ for cooperative communication in cellular networks and the scheduling algorithm is called proportionally fair selective cooperation (PFSC).

The paper is structured as follows. In Section 2, we first describe the assumptions made in our study of the problem and the resulting problem formulations, we then propose the PFSC algorithm that maximizes the overall utility of a cooperative cellular network. After that, analytical results of PFSC are presented in Section 3. Finally, in Section 4, we present simulation results to validate the theoretical model and throughput performance of PFSC, followed by the conclusion in Section 5.

\section{System Model}

For an $N$-node TDMA cellular network, let's consider the problem where these $N$ nodes wishing to transmit data to the BS. We assume that each node can function as a DF relay as needed and single-input-single-output (SISO) is used for communications.

\subsection{Problem Formulation}

As shown in Fig. 1, unlike the BS in a traditional cellular network where only one node is selected for transmission at a time, the BS in the considered network schedules a pair of nodes $(m, r)$ at each slot. Once the pair $(m, r)$ is selected, Node $m$ will transmit and Node $r$ will function as a DF relay. If Node $r$ successfully decodes the signal received from Node $m$, it will relay the signal to the BS. The BS will decode using the signals received from nodes $m$ and $r$.

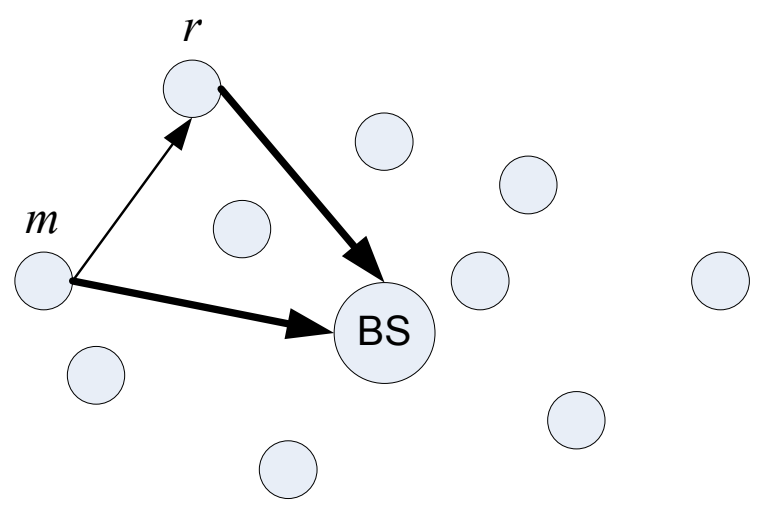

\section{$r$ : Mobile node acting as relay $\longrightarrow$ : Non-cooperative link $m$ : Mobile node $\longrightarrow$ : Cooperative link}

Fig. 1. 2-node cooperative cellular network 
We use $\Theta$ to denote the set of nodes in the network and $\Omega=\{(m, r) \mid m, r \in$ $\Theta, m \neq r\}$ to denote the set of all possible node pairs. Obviously, for an $N$ node cellular network, there are ${ }_{N} P_{2}=N \times(N-1)$ possible $(m, r)$ pairs. We use $m \rightarrow r,(n, r) \rightarrow B S$ to denote the non-cooperative link and the cooperative link, respectively. According to Fig. 1, a transmission over a non-cooperative link together with a transmission over the corresponding cooperative link compose a relay-aided 2-node transmission. Let $R_{m, r}^{N C}[t+1], R_{m, r}^{C}[t+1]$ be the instantaneous capacities of the non-cooperative link $m \rightarrow r$ and the cooperative link $(m, r) \rightarrow$ $B S$, and $R_{m, r}[t+1]$ be the instantaneous capacity of the corresponding 2-node transmission, in next time slot $t+1 . R_{m, r}[t]$ 's $k$-point moving average throughput (i.e., short-term average throughput) up to time $t$ is denoted by $\mu_{m, r}[t]$.

Because of the superior performance of the PFS algorithm, we apply the PF criterion $[15,21]$ in the cooperative cellular network shown in Fig. 1. Similar to the traditional PFS problem in a cellular network without cooperation, for a 2-node cooperative cellular network with $\mathrm{PF}$ constraint, the objective is to maximize the overall logarithmic utility of all 2-node transmission. This is formulated as follows

$$
\max \sum_{(m, r) \in \Omega} \ln \left(\mu_{m, r}(t)\right) .
$$

given,

$$
\begin{gathered}
I_{m, r}[t+1]= \begin{cases}1 & \text { if node pair }(m, r) \in \Omega \text { is scheduled } \\
0 & \text { else }\end{cases} \\
\mu_{m, r}[t+1]=\left(1-\frac{1}{k}\right) \mu_{m, r}[t]+I_{m, r}[t+1] \times \frac{R_{m, r}[t+1]}{k} . \\
R_{m, r}[t+1]=\min \left(R_{m, r}^{N C}[t+1], R_{m, r}^{C}[t+1]\right) .
\end{gathered}
$$

(2) indicates that at each time slot, only one node and one accompanying relay are selected for cooperative transmission at next time slot. The min operation in (4) accounts for the successful decoding required at both Node $r$ and the BS.

\subsection{Proportionally Fair Selective Cooperation (PFSC)}

For the above optimization problem, we propose the following solution, namely the proportionally fair selective cooperation (PFSC) algorithm.

As pointed out earlier, it has been proved that PFS maximizes the overall logarithmic utility of a cellular network by scheduling the Node $j$ having the maximum PF metric defined as the ratio of the instantaneous rate to its short-term average throughput tracked by an exponential moving average [15]. Similarly, PFSC maximizes the overall logarithmic utility of a 2-node cooperative cellular network by scheduling the node pair $(m, r)$ having the maximum PFSC metric $\frac{R_{m, r}[t+1]}{\mu_{m, r}[t]}$. In other words, the proposed PFSC algorithm is the optimal solution of (1). 


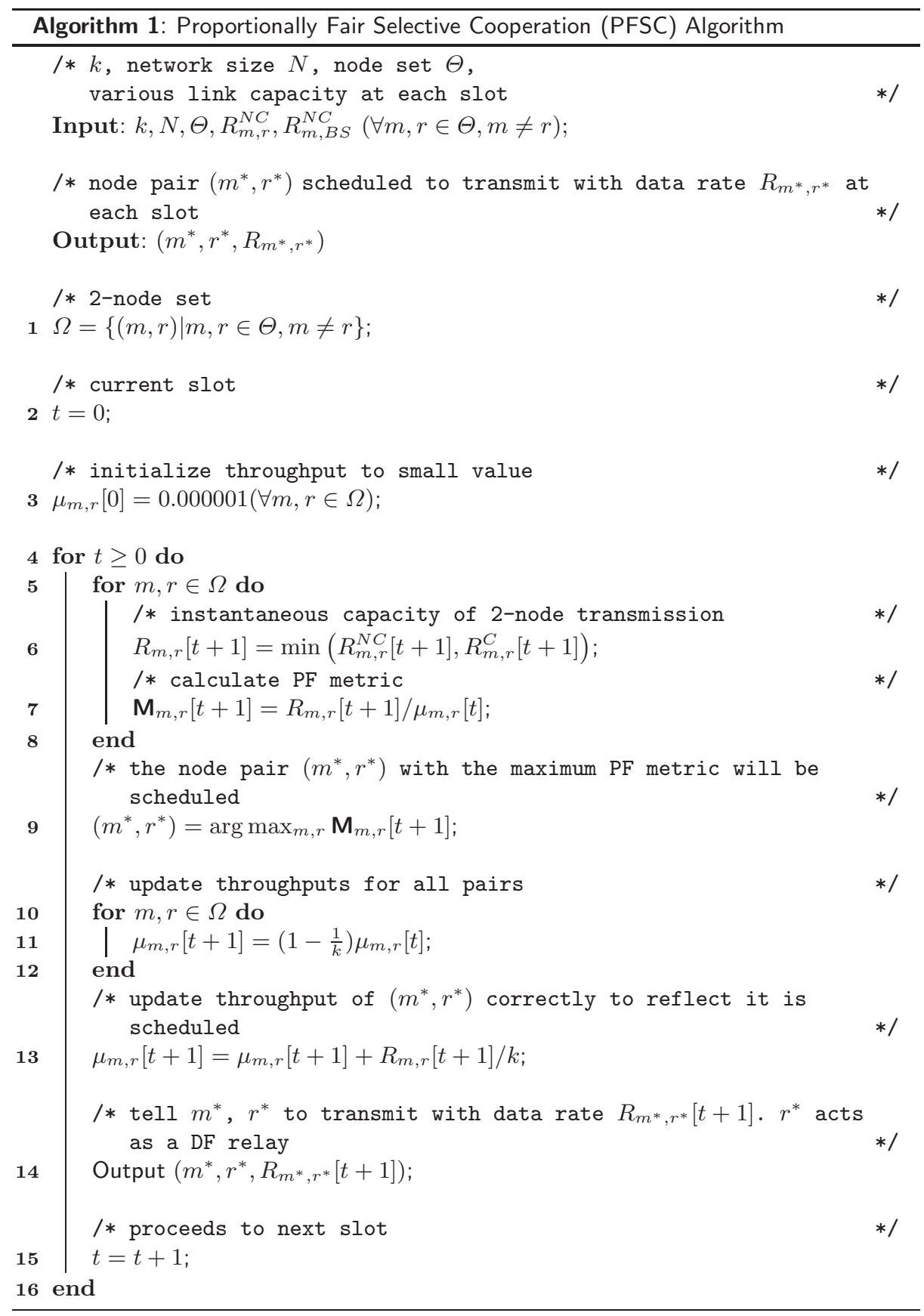

To implement PFSC, the BS needs to know the channel state information (CSI) of both the $m \rightarrow B S$ and $m \rightarrow r$ links. Relay needs to know the CSI of 
the $m$ to relay link, which will be sent to the BS. We assume that the network operates in a slow fading scenario, so that channel estimation is possible and CSI is available at both the BS and relays.

The differences between PFSC and PFS are: in an $N$-node cellular network, with PFS the BS will schedule $N$ nodes while with PFSC the BS will schedule $N \times(N-1)$ "nodes" (i.e., node pairs); with PFS, the instantaneous capacity of Node $m$ is estimated by the link quality of $m \rightarrow B S$, while with PFSC, the instantaneous capacity of node pair $(m, r)$ should be estimated by the link quality of both $m \rightarrow r$ and $(m, r) \rightarrow B S$.

\section{Analysis of PFSC over a Rayleigh Flat Fading Channel}

In this section, we analyze the proposed PFSC algorithm over Rayleigh flat fading channels, and provide closed-form theoretical results. For the analysis, we use the following assumptions:

- Both the non-cooperative link and the cooperative link are Rayleigh flat faded;

- The fading between any different links (no matter they are non-cooperative links or cooperative links) is mutually independent but not necessarily identical distributed;

- Channel fading keeps constant over each slot, and varies independently from slot to slot;

- Maximal ratio combining (MRC) is used at the BS to combine the signals from the source and relay nodes..

To include the distance-dependent path loss, we use the model seen in [4], i.e.,, the instantaneous SNR is the multiplication of the average SNR (determined by path loss and shadowing ${ }^{2}$ ) with an randomly fading variable. This results in a single random variable that jointly models path loss and fading. Consequently, the instantaneous SNR of a Rayleigh fading link is modeled as an exponentially distributed random variable with an average SNR (in dB) given by

$$
\overline{S N R_{d}}=\overline{S N R_{d_{0}}}-\alpha \times 10 \log _{10}\left(d / d_{0}\right) .
$$

where $\alpha$ is path loss exponent, $d$ denotes link distance, and $\overline{S N R_{d_{0}}}$ is the average SNR at reference distance $d_{0}$.

Refer to Fig. 1, we use $S N R_{m, r}^{N C}, S N R_{m, r}^{C}$ to denote the instantaneous SNRs of the non-cooperative link $m \rightarrow r$ and the cooperative link $(m, r) \rightarrow B S$, respectively.

We use $\overline{S N R_{m, r}^{N C}}, \overline{S N R_{m, B S}^{N C}}, \overline{S N R_{r, B S}^{N C}}$ to denote the mean SNRs of the noncooperative links $m \rightarrow r, m \rightarrow B S$, and $r \rightarrow B S$, respectively.

Due to Rayleigh fading, the instantaneous SNR of a non-cooperative link is an exponentially distributed random variable with a probability density function (pdf) given by

\footnotetext{
${ }^{2}$ Without loss of generality, shadowing is not considered here.
} 


$$
\rho_{i, j}^{N C}(x)=\frac{1}{\overline{S N R_{i, j}^{N C}}} \times e^{-x / \overline{S N R_{i, j}^{N C}}}, \forall(i, j) \in \Theta, \text { or } j=B S .
$$

With MRC, the combined SNR is the sum of the SNRs of each individual diversity branch. The pdf of the instantaneous SNR of a cooperative link is then given by

$$
\rho_{m, r}^{C}(x)=\frac{1}{\overline{S N R_{m, B S}^{N C}}+\overline{S N R_{r, B S}^{N C}}} \times e^{-x /\left(\overline{S N R_{m, r}^{N C}}+\overline{S N R_{r, B S}^{N C}}\right)}
$$

Unless otherwise specified, system bandwidth is normalized to $1 \mathrm{~Hz}$. Using Shannon formula, we can write the capacities of the non-cooperative link and the cooperative link as follows

$$
\begin{aligned}
& R_{m, r}^{N C}=\log _{2}\left(1+S N R_{m, r}^{N C}\right) . \\
& R_{m, r}^{C}=\log _{2}\left(1+S N R_{m, r}^{C}\right) .
\end{aligned}
$$

Substituting (8) and (9) into (4), we have

$$
\begin{aligned}
R_{m, r}[t+1] & =\min \left(R_{m, r}^{N C}[t+1], R_{m, r}^{C}[t+1]\right) \\
& =\log _{2}\left(1+\min \left(S N R_{m, r}^{N C}[t+1], S N R_{m, r}^{C}[t+1]\right)\right) .
\end{aligned}
$$

We know that the distribution of the minimum of multiple independently exponential random variables is also an exponential distributed random variable, i.e., for $n$ independently exponential distributed random variables $g_{1}, g_{2}, \ldots, g_{n}$ with parameters $\lambda_{1}, \lambda_{2}, \ldots, \lambda_{n}, g=\min \left\{g_{1}, g_{2}, \ldots, g_{n}\right\}$ is also exponentially distributed, with parameter

$$
\lambda=\lambda_{1}+\lambda_{2}+\ldots+\lambda_{n}
$$

We then have the capacity of the 2-node transmission,

$$
R_{m, r}[t+1]=\log _{2}\left(1+S N R_{m, r}[t+1]\right) .
$$

where $S N R_{m, r}$ is an exponentially distributed random variable with a pdf given by

$$
\begin{aligned}
\rho_{m, r}(x)= & \left(\frac{1}{\overline{S N R_{m, B S}^{N C}}+\overline{S N R_{r, B S}^{N C}}}+\frac{1}{\overline{S N R_{m, r}^{N C}}}\right) \\
& \quad-\left(\frac{x}{\overline{S N R_{m, B S}^{N C}}+\overline{S N R_{r, B S}^{N C}}}+\frac{x}{\overline{S N R_{m, r}^{N C}}}\right) .
\end{aligned}
$$

(12) indicates that $S N R_{m, r}$ represents the effective SNR of the 2-node transmission $\{m \rightarrow r,(m, r) \rightarrow B S\}$. By (13), we obtain the mean value of $S N R_{m, r}$ 


$$
\overline{S N R_{m, r}}=\frac{1}{\left(\frac{1}{\overline{S N R_{m, B S}^{N C}}+\overline{S N R_{r, B S}^{N C}}}+\frac{1}{\overline{S N R_{m, r}^{N C}}}\right)} .
$$

[28] and [29] have shown that, in Rayleigh or Ricean fading networks, the Shannon capacity $R=\log _{2}[1+S N R]$ can be accurately approximated by Normal distribution. Specially, for a SISO link over a Rayleigh flat fading channel where SNR is exponential distributed, the Normal distribution is characterized by a mean and variance given as follows

$$
\begin{gathered}
E[R]=\int_{0}^{\infty} e^{-x} \times \log _{2}(1+\overline{S N R} \times x) d x \\
\sigma^{2}=\int_{0}^{\infty} e^{-x} \times\left(\log _{2}(1+\overline{S N R} \times x)\right)^{2} d x-(E[R])^{2} .
\end{gathered}
$$

According to the above discussion, we have the following theorem.

Theorem 1. In a Rayleigh flat fading cellular network with 2-node cooperation, the capacity of a 2-node transmission can be characterized by Normal distribution.

Specifically, with (12)-(16), we obtain the Normal distribution that characterizes the capacity of the 2-node transmission $\{m \rightarrow r,(m, r) \rightarrow B S\}$

$$
\begin{aligned}
E\left[R_{m, r}\right]= & \int_{0}^{\infty} e^{-x} d x \times \log _{2}\left(1+\frac{x}{\left(\frac{1}{\overline{S N R_{m, B S}^{N C}}+\overline{S N R_{r, B S}^{N C}}}+\frac{1}{\overline{S N R_{m, r}^{N C}}}\right)}\right) \\
\sigma_{m, r}^{2}= & \int_{0}^{\infty} e^{-x} d x \times\left(\log _{2}\left(1+\frac{x}{\left(\frac{1}{\overline{S N R_{m, B S}^{N C}}+\overline{S N R_{r, B S}^{N C}}}+\frac{1}{\overline{S N R_{m, r}^{S R}}}\right)}\right)\right)^{2} \\
& -\left(E\left[R_{m, r}\right]\right)^{2}
\end{aligned}
$$

According to our previous research $[21,30]$, we have the following lemma.

Lemma 1 (PFS Throughput). For a PFS-enabled $N$-node cellular network, if the instantaneous capacity is a Normally distributed random variable, the longterm average throughput of Node $i$ is given by

$$
E\left[\mu_{i}\right]=\frac{E\left[R_{i}\right]}{N} \times\left(1-\left[\phi\left(-M_{i}\right)\right]^{N}\right)+\int_{-M_{i}}^{\infty} y \sigma_{i} \rho(y) \times[\phi(y)]^{N-1} d y .
$$

where $R_{i}$ and $\mu_{i}$ are the instantaneous capacity and throughput of Node $i$, $E\left[R_{i}\right]$ and $\sigma_{i}$ denote the statistical average and standard deviation of $R_{i}$, $M_{i}=E\left[R_{i}\right] / \mu_{i}, \rho(\cdot)$ and $\phi(\cdot)$ are the pdf and cdf of zero mean, unit variance standard normal distribution. 
According to Theorem 1, we know that in a Rayleigh flat fading environment, the capacity of a node pair in a PFSC-enabled cellular network can be modeled by Normal distribution. With Theorem 1 and Lemma 1, we have the following important theoretical theorem for PFSC.

Theorem 2. For a Rayleigh fading PFSC-enabled cellular network, the longterm average throughput of Node $m$ is given by

$$
\begin{aligned}
E\left[\mu_{m}\right]= & \sum_{r \in \Theta, r \neq m} E\left[\mu_{m, r}\right] \\
= & \sum_{r \in \Theta, r \neq m} \frac{E\left[R_{m, r}\right]}{N(N-1)} \times\left(1-\left[\phi\left(-M_{m, r}\right)\right]^{N(N-1)}\right) \\
& +\sum_{r \in \Theta, r \neq m} \int_{-M_{m, r}}^{\infty} y \sigma_{m, r} \rho(y) \times[\phi(y)]^{N(N-1)-1} d y .
\end{aligned}
$$

where $R_{m, r}$ and $\mu_{m, r}$ are the instantaneous capacity and throughput of node pair $(m, r), E\left[R_{m, r}\right]$ and $\sigma_{m, r}$ denote the statistical average and standard deviation of $R_{m, r}, M_{m, r}=E\left[R_{m, r}\right] / \mu_{m, r}$.

Proof. There are ${ }_{N} P_{2}=N(N-1)$ possible node pairs in an $N$-node cellular network. In the beginning of this section, we have proved that in a Rayleigh fading cellular network with 2-node cooperation, the capacity $E\left[\mu_{m, r}\right]$ of a node pair $(m, r)$ can be modeled as a Normally distributed random variable (Theorem 1 ). Viewing a pair in a PFSC-enabled cellular network as a node in a PFS-enabled cellular network, and applying Lemma 1, we have

$$
\begin{aligned}
E\left[\mu_{m, r}\right]= & \frac{E\left[R_{m, r}\right]}{N(N-1)} \times\left(1-\left[\phi\left(-M_{m, r}\right)\right]^{N(N-1)}\right) \\
& +\int_{-M_{m, r}}^{\infty} y \sigma_{m, r} \rho(y) \times[\phi(y)]^{N(N-1)-1} d y .
\end{aligned}
$$

where $E\left[R_{m, r}\right]$ and $\sigma_{m, r}$ are given by (17) and (18).

Since $E\left[\mu_{m}\right]=\sum_{r \in \Theta, r \neq m} E\left[\mu_{m, r}\right]$, Theorem 2 follows immediately.

With Theorem 2 and Lemma 1, we have the following corollary.

Corollary 1. For Node $m$ in a Rayleigh fading cellular network, the throughput gain of PFSC over PFS is

$$
G_{m}=\frac{\sum_{r \in \Theta, r \neq m}\left(\begin{array}{l}
\frac{E\left[R_{m, r}\right]}{N(N-1)} \times\left(1-\left[\phi\left(-M_{m, r}\right)\right]^{N(N-1)}\right. \\
+\int_{-M_{m, r}}^{\infty} y \sigma_{m, r} \rho(y) \times[\phi(y)]^{N(N-1)-1} d y
\end{array}\right)}{\left(\frac{E\left[R_{m}\right]}{N} \times\left(1-\left[\phi\left(-M_{m}\right)\right]^{N}\right)+\int_{-M_{m}}^{\infty} y \sigma_{m} \rho(y) \times[\phi(y)]^{N-1} d y\right)} .
$$

where $R_{m, r}$ and $\mu_{m, r}$ are the instantaneous capacity and throughput of node pair $(m, r), E\left[R_{m, r}\right]$ and $\sigma_{m, r}$ denote the statistical average and standard deviation of $R_{m, r}, M_{m, r}=E\left[R_{m, r}\right] / \mu_{m, r}$. 
Remark 1. Numerical calculations show that $\left(\phi\left(-M_{m}\right)\right)^{N} \ll 1,\left(\phi\left(-M_{m, r}\right)\right)^{N(N-1)} \ll$ $1, \int_{-M_{m}}^{\infty} y \rho(y)[\phi(y)]^{N-1} d y \approx \int_{0}^{\infty} y \rho(y)[\phi(y)]^{N-1} d y, \int_{-M_{m}, r}^{\infty} y \rho(y)[\phi(y)]^{N(N-1)-1} d y \approx$ $\int_{0}^{\infty} y \rho(y)[\phi(y)]^{N(N-1)-1} d y$, so Theorem 2 and Corollary 1 reduce to the following forms,

$$
\begin{gathered}
E\left[\mu_{m, r}\right]=\frac{E\left[R_{m, r}\right]}{N(N-1)}+\sigma_{m, r} \int_{0}^{\infty} y \rho(y)[\phi(y)]^{N(N-1)} d y . \\
G_{m}=\frac{\sum_{r \in \Theta, r \neq m}\left(\frac{E\left[R_{m, r}\right]}{N(N-1)}+\sigma_{m, r} \int_{0}^{\infty} y \rho(y)[\phi(y)]^{N(N-1)-1} d y\right)}{\left(\frac{E\left[R_{m}\right]}{N}+\sigma_{m} \int_{0}^{\infty} y \rho(y)[\phi(y)]^{N-1} d y\right)} .
\end{gathered}
$$

Corollary 1 provides a closed-form expression for quantifying the throughput gain with 2-node cooperation for cellular networks with PF constraint.

\section{Numerical and Simulation Results}

We first performed numerical experiment to evaluate the throughput performance of PFSC algorithm over a Rayleigh flat fading channel.

\subsection{Numerical Experiment}

In the numerical experiment, we use the following settings: As shown in Fig. 2, 10 nodes $n_{1} \sim n_{10}$ are placed in an area of $1.2 \mathrm{~km} \times 1.6 \mathrm{~km}$. Path loss exponent $\alpha=2.5 \sim 4.5$ for various environment, reference distance $d_{0}=100 \mathrm{~m}$, reference SNR at $d_{0}$ is $S N R_{d_{0}}=30 \mathrm{~dB}$.

Equation (5) is used to calculate the SNR (in $\mathrm{dB}$ ) at distance $d$. After that, (15) and (16) are used to calculate the mean and standard deviation of the capacity for each node; (14), (17) and (18) are used to calculate the mean and standard deviation of the capacity for each $(m, r)$ pair. Finally, we use $(20)$ or (22) to evaluate the performance of PFSC.

We plot in Fig. 3 the throughput gain $G_{m}$ of PFSC over PFS for each Node $m$ under various path loss scenarios. We can see that with node cooperation, PFSC significantly improves performance. Fig. 3 also shows that the PFSC performance increases with path loss exponent and bad-channel nodes $n_{1}, n_{2}, n_{3}, n_{10}$ benefit more from PFSC than good-channel nodes $n_{4} \sim n_{9}$. These indicate that PFSC will perform better in relatively bad communication environment.

In Fig. 3, we notice Node $n_{5}$ has relatively small gain. This is due to two reasons: 1). Node $n_{5}$ locates near to the BS and already has relatively good channel condition. This makes relaying less efficient; 2). All possible DF relays are far from Node $n_{5}$. This means that the received SNRs may not be high enough to allow successful decoding at the DF relays, which in turn reduces the relaying gain; 


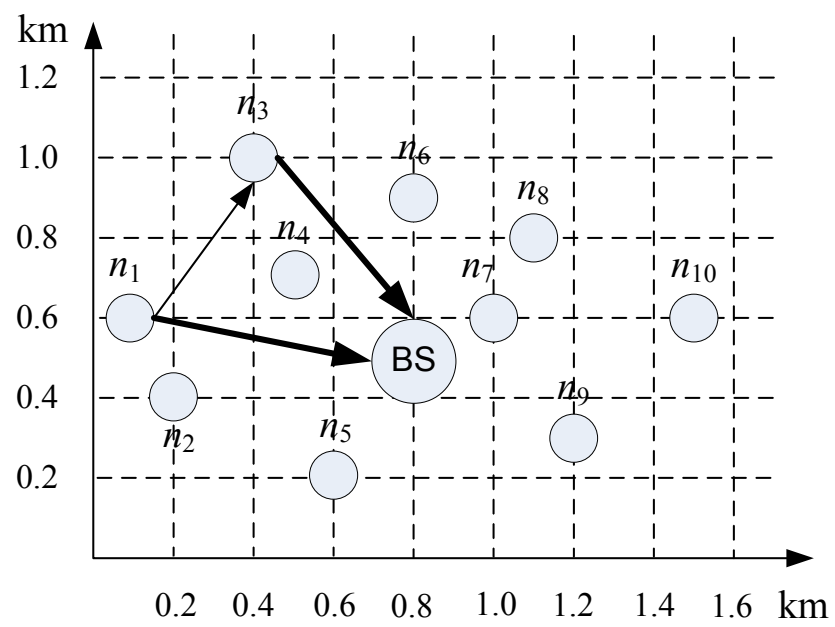

Fig. 2. Network Topology: a 10-node Cellular Network

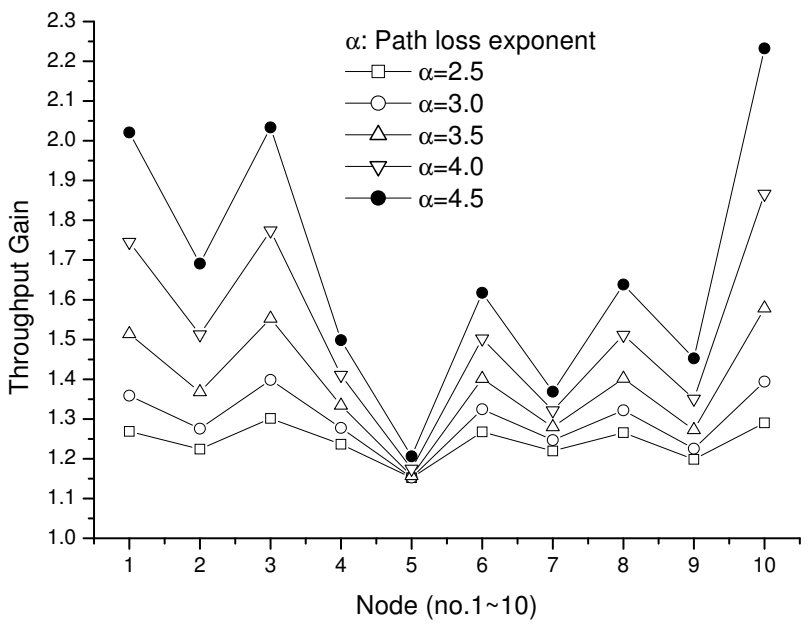

Fig. 3. PFSC v.s. PFS: a throughput comparison

\subsection{Simulation}

We now evaluate the accuracy of our theoretical models by simulation. We use the same network topology as shown in Fig. 2. Each link undergoes independent Rayleigh flat fading. The PFSC algorithm presented in Subsection 2.2 is used in simulation. Path loss exponent is 3.0. Moving average factor $k=500$. The simulation runs for 8000 slots. Fig. 4 depicts the curves from simulation and theoretical results for nodes $n_{1}, n_{7}$, and $n_{9}$. Node $n_{1}$ is the farthest node from the BS and Node $n_{7}$ is the nearest one. Nodes $n_{1}, n_{9}$, and $n_{7}$ represent the worst-, the medium-, and the best-channel nodes in a cellular network. 


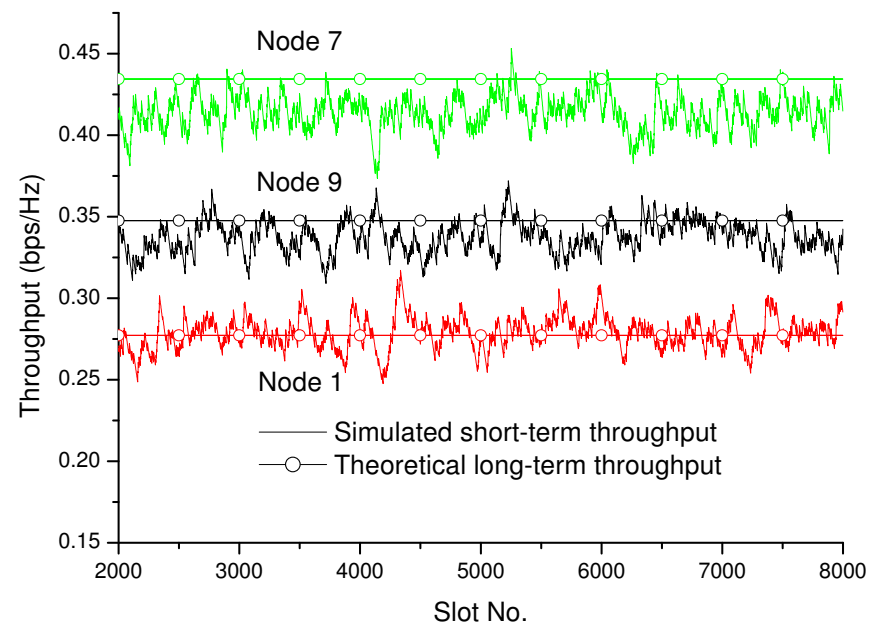

Fig. 4. PFSC for the worst/medium/best node: Simulation v.s. Analysis

The validity of our theoretical results is illustrated in Fig. 4 where the solid lines are the short-term throughput from simulation and the circle ones are the long-term throughput from analysis. We can see that our theoretical analysis matches well with simulations for all three nodes with $\sim 98 \%$ accuracy.

\section{Conclusion}

2-node selective cooperation in cellular networks is a practical cooperation method that does not require complex code design to benefit from the spatial diversity. Adding proportional fairness into 2-node selective cooperation, we propose the proportionally fair selective cooperation (PFSC) algorithm for cellular networks. This technique integrates well-known proportional fairness with traditional MRC reception and thus allows for immediate implementation in existing hardware.

To facilitate researches, we further analyzed PFSC and presented a framework to quantify the performance of PFSC. Numerical results validates that PFSC significantly improves the throughput performance, and the simulation shows the accuracy of our analysis.

The increased performance of PFSC over PFS comes with additional overhead: To implement PFSC, the BS needs to collect the CSI of both $m$ to the BS and $m$ to relay links. Relay needs to know the CSI of the $m$ to relay link, which is sent to the BS via a control/feedback channel. Even with this overhead, PFSC is still a promising solution for efficient data communications in a cooperative cellular network, due to its simplicity and superior performance in terms of throughput. 


\section{References}

1. A. Sendonaris, E. Erkip, and B. Aazhang, "User cooperation diversity. part i. system description," IEEE Transactions on Communications, vol. 51, no. 11, pp. 1927-1938, November 2003.

2. — - "User cooperation diversity. part ii. implementation aspects and performance analysis," IEEE Transactions on Communications, vol. 51, no. 11, pp. 1939-1948, November 2003.

3. T. C.-Y. Ng and W. Yu, "Joint optimization of relay strategies and resource allocations in cooperative cellular networks," IEEE Journal on Selected Areas in Communications, vol. 25, no. 2, pp. 328-339, 2007.

4. J. N. Laneman, D. N. C. Tse, and G. W. Wornell, "Cooperative diversity in wireless networks: Efficient protocols and outage behavior," IEEE Transactions on Information Theory, vol. 50, no. 12, pp. 3062-3080, 2004.

5. E. Aktas, J. Evans, and S. Hanly, "Distributed decoding in a cellular multipleaccess channel," IEEE Transactions on Wireless Communications, vol. 7, no. 1, pp. 241-250, 2008.

6. A. Bletsas, A. Khisti, D. P. Reed, and A. Lippman, "A simple cooperative diversity method based on network path selection," IEEE Journal on Selected Areas in Communications, vol. 24, no. 3, pp. 659-672, March 2006.

7. Y. Sung, S. Misra, L. Tong, and A. Ephremides, "Cooperative routing for distributed detection in large sensor networks," IEEE Journal on Selected Areas in Communications, vol. 25, no. 2, pp. 471-483, 2007.

8. J. Huang, Z. Han, M. Chiang, and H. V. Poor, "Auction-based resource allocation for cooperative communications," IEEE Journal on Selected Areas in Communications, vol. 26, no. 7, pp. 1226-1237, 2008.

9. O. Simeone, O. Somekh, G. Kramer, H. V. Poor, and S. Shamai, "Throughput of cellular systems with conferencing mobiles and cooperative base stations," EURASIP Journal on Wireless Communications and Networking, vol. 2008, pp. 1-14, 2008.

10. W. Chen, L. Dai, K. Ben Letaief, and Z. Cao, "A unified cross-layer framework for resource allocation in cooperative networks," IEEE Transactions on Wireless Communications, vol. 7, no. 8, pp. 3000-3012, 2008.

11. A. Papadogiannis, E. Hardouin, and D. Gesbert, "Decentralising multi-cell cooperative processing on the downlink : a novel robust framework," EURASIP Journal on Wireless Communications and Networking, August 2009.

12. E. Liu, Q. Zhang, and K. K. Leung, "Theoretical analysis of selective relaying, cooperative multi-hop networks with fairness constraints," in Proc. IEEE International Conference on Communications ICC 2009, June 2009, pp. 1-5.

13. A. D. Coso, U. Spagnolini, and C. Ibars, "Cooperative distributed MIMO channels in wireless sensor networks," IEEE Journal on Selected Areas in Communications, vol. 25, no. 2, pp. 402-414, 2007.

14. Y. Rong and Y. Hua, "Optimal power schedule for distributed MIMO links," IEEE Transactions on Wireless Communications, vol. 7, no. 8, pp. 2896-2900, 2008.

15. F. Kelly, "Charging and rate control for elastic traffic," European Transactions on Telecommunications, vol. 8, pp. 33-37, February 1997.

16. H. J. Kushner and P. A. Whiting, "Convergence of proportional-fair sharing algorithms under general conditions," IEEE Transactions on Wireless Communications, vol. 3, no. 4, pp. 1250-1259, 2004.

17. S. Borst, "User-level performance of channel-aware scheduling algorithms in wireless data networks," IEEE/ACM Transactions on Networking, vol. 13, no. 3, pp. 636-647, June 2005. 
18. T. Bu, L. Li, and R. Ramjee, "Generalized proportional fair scheduling in third generation wireless data networks," in Proc. INFOCOM 2006. The 25th Joint Conference of the IEEE Computer and Communications Societies. IEEE, April 2006, pp. $1-12$.

19. G. Caire, R. R. Muller, and R. Knopp, "Hard fairness versus proportional fairness in wireless communications: The single-cell case," IEEE Transactions on Information Theory, vol. 53, no. 4, pp. 1366-1385, April 2007.

20. L. Li, M. Pal, and Y. R. Yang, "Proportional fairness in multi-rate wireless lans," in Proc. INFOCOM 2008. The 27th Joint Conference of the IEEE Computer and Communications Societies. IEEE, April 2008, pp. 1004-1012.

21. E. Liu and K. K. Leung, "Expected throughput of the proportional fair scheduling over Rayleigh fading channels," IEEE Communications Letters, vol. 14, no. 6, pp. 515-517, 2010.

22. G. Bianchi and A. T. Campbell, "A programmable mac framework for utility-based adaptive quality of service support," IEEE Journal on Selected Areas in Communications, vol. 18, no. 2, pp. 244-255, 2000.

23. L. Shi, C. Liu, and B. Liu, "Network utility maximization for triple-play services," Computer Communications, vol. 31, no. 10, pp. 2257-2269, 2008.

24. D. Xu, Y. Li, M. Chiang, and A. Calderbank, "Elastic service availability: utility framework and optimal provisioning," IEEE Journal on Selected Areas in Communications, vol. 26, no. 6, pp. 55-65, 2008.

25. X. Zhang, M. Tao, and C. Ng, "Utility-based wireless resource allocation for variable rate transmission," IEEE Transactions on Wireless Communications, vol. 7, no. 9, pp. 3292-3296, 2008.

26. Q. Gao, J. Zhang, and S. Hanly, "Cross-layer rate control in wireless networks with lossy links: Leaky-pipe flow, effective network utility maximization and hop-by-hop algorithms," IEEE Transactions on Wireless Communications, vol. 8, no. 6, pp. 3068-3076, 2009.

27. A. Jalali, R. Padovani, and R. Pankaj, "Data throughput of CDMA-HDR a high efficiency-high data rate personal communication wireless system," in Proc. IEEE 51st VTC 2000-Spring Tokyo Vehicular Technology, vol. 3, January 2000, pp. 18541858.

28. P. J. Smith, S. Roy, and M. Shafi, "Capacity of MIMO systems with semicorrelated flat fading," IEEE Transactions on Information Theory, vol. 49, no. 10, pp. 27812788, October 2003.

29. M. R. McKay, P. J. Smith, H. A. Suraweera, and I. B. Collings, "On the mutual information distribution of OFDM-based spatial multiplexing: Exact variance and outage approximation," IEEE Transactions on Information Theory, vol. 54, no. 7, pp. 3260-3278, July 2008.

30. E. Liu, Q. Zhang, and K. K. Leung, "Resource allocation for frequency-selective fading, multi-carrier systems," in Proc. IEEE International Conference on Communications ICC 2009, June 2009. 